\title{
2015 ESC guidelines for the management of acute coronary syndromes in patients presenting without persistent ST-segment elevation: comments from the Dutch ACS working group
}

\author{
P. Damman' ${ }^{1}$ A. W. van 't Hof ${ }^{2}$ J. M. ten Berg ${ }^{3} \cdot$ J. W. Jukema ${ }^{4}$ Y. Appelman ${ }^{5}$ A. H. Liem ${ }^{6}$ R. J. de Winter'1
}

Published online: 13 December 2016

(C) The Author(s) 2016. This article is available at SpringerLink with Open Access.

\begin{abstract}
On behalf of the Dutch ACS working group, we discuss multiple recommendations which have been implemented in the 2015 ESC guidelines for the management of acute coronary syndromes (ACS) in patients presenting without persistent ST-segment elevation.
\end{abstract}

Keywords NSTE-ACS guidelines - NVVC ACS working group statement

\section{Introduction}

The 2015 ESC guidelines for the management of acute coronary syndromes (ACS) in patients presenting without persistent ST-segment elevation were presented at the European Society of Cardiology (ESC) Conference 2015 in London and published in the European Heart Journal [1]. Compared with the 2011 version, multiple recommendations

P. Damman, A.W. van 't Hof, J.M. ten Berg, J.W. Jukema, Y. Appelman, A.H. Liem and R.J. de Winter represent the NVVC Acute Coronary Syndrome working group

R. J. de Winter

r.j.dewinter@amc.uva.nl

1 Academic Medical Center, University of Amsterdam, Amsterdam, The Netherlands

2 Isala Klinieken, Zwolle, The Netherlands

3 St Antonius Hospital Nieuwegein, Nieuwegein, The Netherlands

4 Leiden University Medical Center, Leiden, The Netherlands

5 VU University Medical Center, Amsterdam, The Netherlands

6 St Franciscus Gasthuis Rotterdam, Rotterdam, The Netherlands have been implemented which we discuss from a Dutch perspective.

\section{High-sensitive troponin}

The introduction of high-sensitive cardiac troponin has led to a better detection and quantification of myocardial injury. Both the absolute value and change in troponin over time provide information on cardiomyocyte injury, and several studies have assessed the sensitivity and specificity of these measurements [2]. In the 2015 guidelines, algorithms are presented for rule-in and rule-out of non-ST-elevation myocardial infarction (NSTEMI) with the use of high-sensitive cardiac troponin (Figs. 1 and 2). We advise to use these high-sensitive troponin assays and incorporate the aforementioned algorithms in daily practice in the Netherlands.

\section{Platelet aggregation inhibition at admission}

When NSTE-ACS is diagnosed, there is an indication for treatment with dual platelet aggregation inhibitors (acetylsalicylic acid and a P2Y12 inhibitor). For patients managed conservatively, the 2015 guidelines advise to use ticagrelor over clopidogrel. While the 2011 ESC guidelines recommended starting dual antiplatelet therapy (DAPT) as soon as possible before coronary angiography [3], the most recent guidelines are less strict suggesting to initiate the P2Y12 inhibitor either before or after coronary angiography. This change is based on the results of the ACCOAST study, in which patients with NSTE-ACS, who were scheduled to undergo catheterisation, were randomised to pretreatment with prasugrel or placebo [4]. Pretreatment with prasugrel did not reduce the rate of major ischaemic events 


\section{Acute Chest Pain}

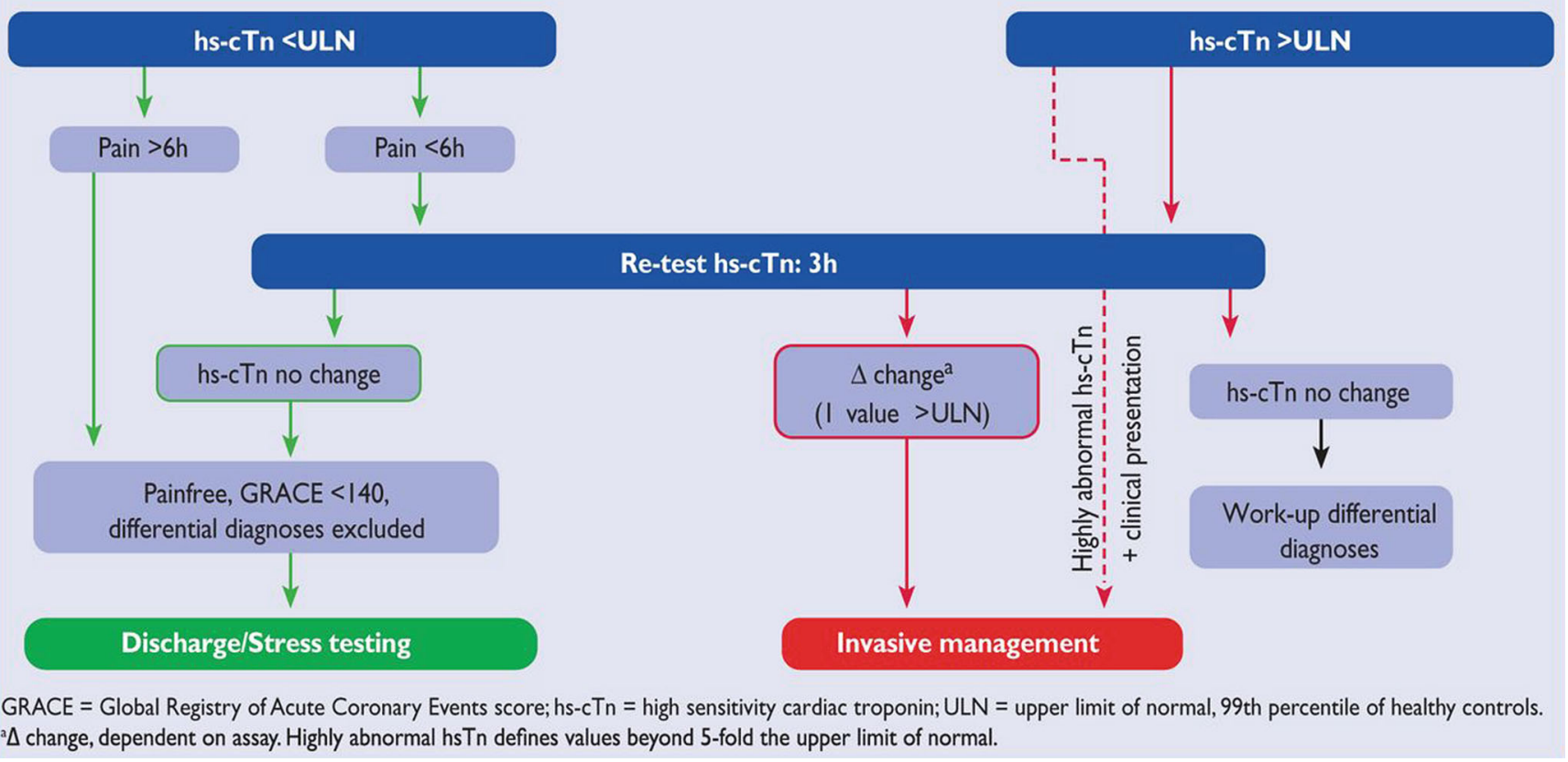

Fig. $10 \mathrm{~h} / 3 \mathrm{~h}$ rule-out algorithms using high-sensitivity cardiac troponin assays in patients presenting to the emergency department with suspected non-ST-elevation myocardial infarction (With permission of Oxford University Press (UK)@ European Society of Cardiology, www.escardio.org)

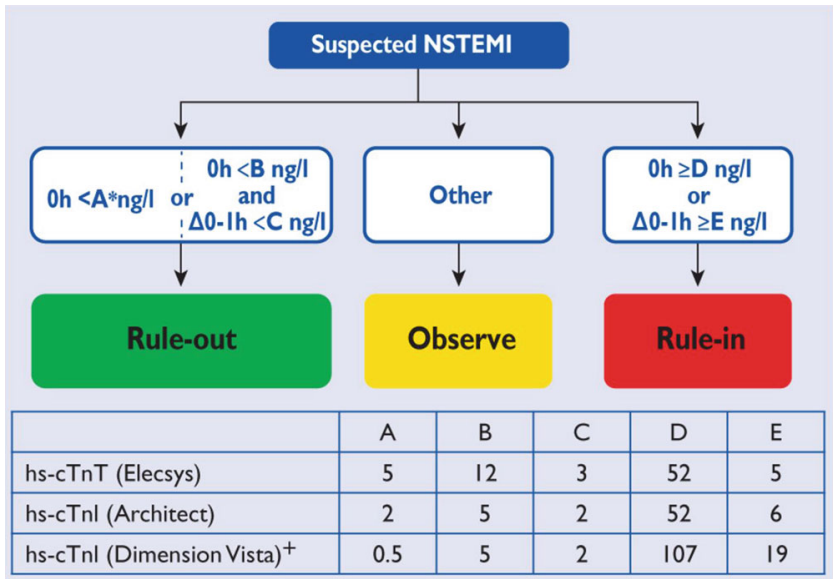

Fig. $20 \mathrm{~h} / 1 \mathrm{~h}$ rule-in and rule-out algorithms using high-sensitivity cardiac troponin assays in patients presenting to the emergency department with suspected non-ST-elevation myocardial infarction (With permission of Oxford University Press (UK)@ European Society of Cardiology, www.escardio.org)

up to 30 days but increased the rate of major bleeding complications. Until more evidence is available, the current guidelines thus provide the opportunity to individualise treatment and postpone the initiation of P2Y12 inhibition in patients with known coronary anatomy or electrocardiographic changes suggesting three-vessel disease or leftmain disease and therefore a suspected indication for early coronary artery bypass surgery (CABG). In patients with a low to intermediate bleeding risk and a high probability of subsequent percutaneous coronary intervention (PCI), pretreatment with clopidogrel or ticagrelor might be useful.

\section{Triple antithrombotic therapy}

A subset of patients with NSTE-ACS have indications for long-term (non-vitamin K) oral anticoagulation ([N]OAC) such as atrial fibrillation or mechanical heart valves. In combination with ACS, regardless of the performance of PCI, there is an indication for triple therapy (DAPT with $[\mathrm{N}] \mathrm{OAC})$. Long-term triple therapy is, however, associated with increased bleeding outcomes [5], and a subsequent increased mortality. Therefore, individualised treatment is necessary in which the ischaemic risk is weighed against the bleeding risk. The current ESC guidelines provide a useful approach in which both the ischaemic and the bleeding risk are taken into account (Fig. 3). In medically managed patients or patients undergoing $\mathrm{CABG}$, a combination of single antiplatelet aggregation therapy and (N)OAC is recommended. If the NSTE-ACS patient undergoes PCI, one or six months of triple therapy is recommended depending on the bleeding risk. After one or six months, a combination of single antiplatelet aggregation therapy and (N)OAC is continued. The Dutch WOEST trial has demonstrated that dual therapy after PCI might be adequate for the pre- 


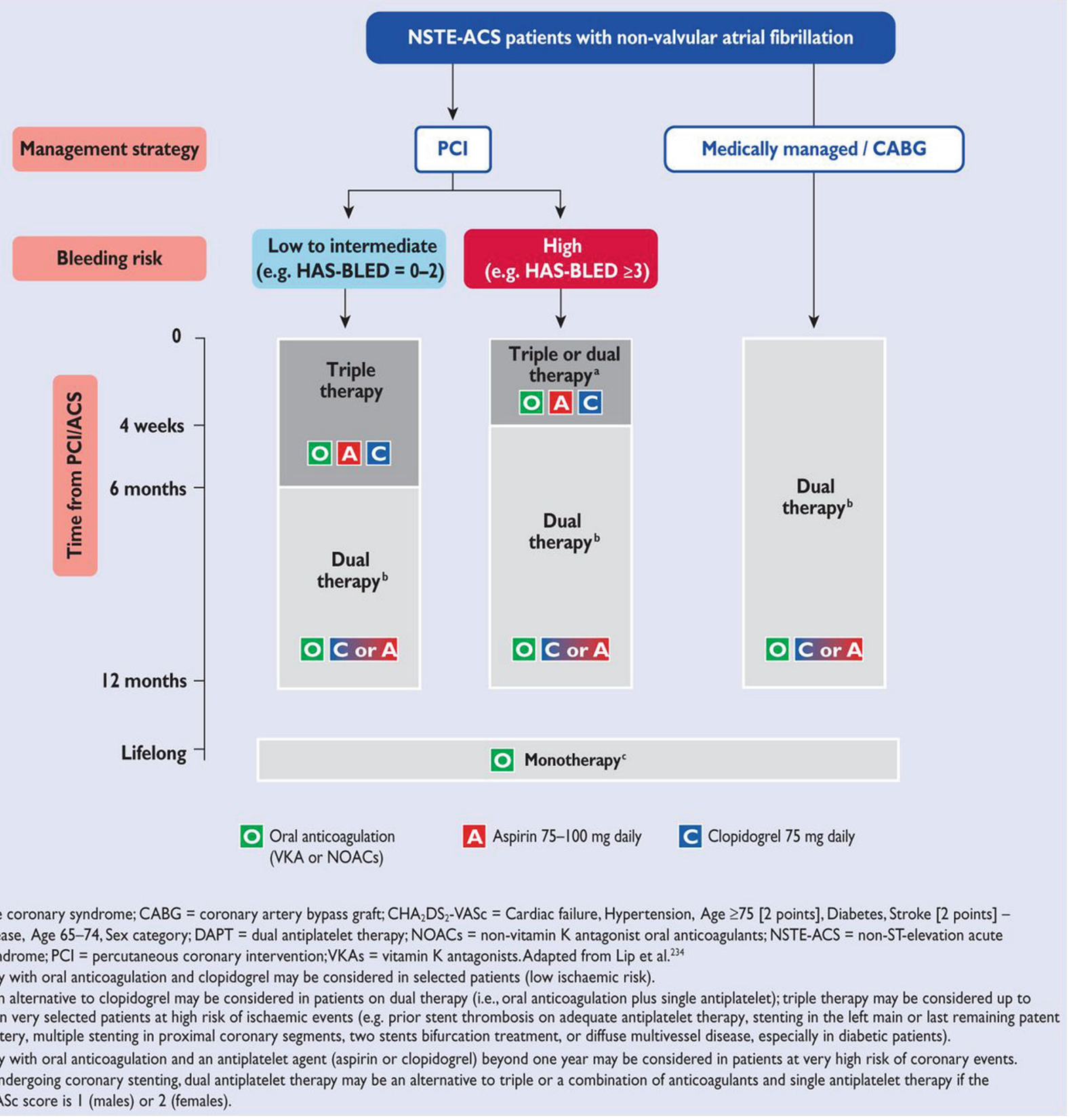

Fig. 3 Antithrombotic strategies in patients with non-ST-elevation acute coronary syndromes and non-valvular atrial fibrillation (With permission of Oxford University Press (UK)@ European Society of Cardiology, www.escardio.org)

vention of ischaemic events, with a reduction of bleeding events [6]. Combinations of (N)OAC with the stronger platelet aggregation drugs prasugrel or ticagrelor is discouraged because of the excessive bleeding risk. We advise to follow the treatment algorithm as shown in Fig. 3, and emphasise to individualise the treatment based on the ischaemic and bleeding risk. Furthermore, there is room for improvement with regards to the communication between the interventional cardiologist performing PCI and the treating physician, especially regarding ischaemic and bleeding risk. Complex coronary interventions, such as multiple stent constructions and the placement of bioabsorbable vascular scaffolds, might require more intensive and longer treatment with DAPT also when combined with (N)OAC. Otherwise, monotherapy with $(\mathrm{N}) \mathrm{OAC}$ is recommended after 1 year.

\section{Same-day transfer in high-risk patients}

Comparable with the 2011 ESC guidelines, the current guidelines mention that the decision for and timing of inva- 


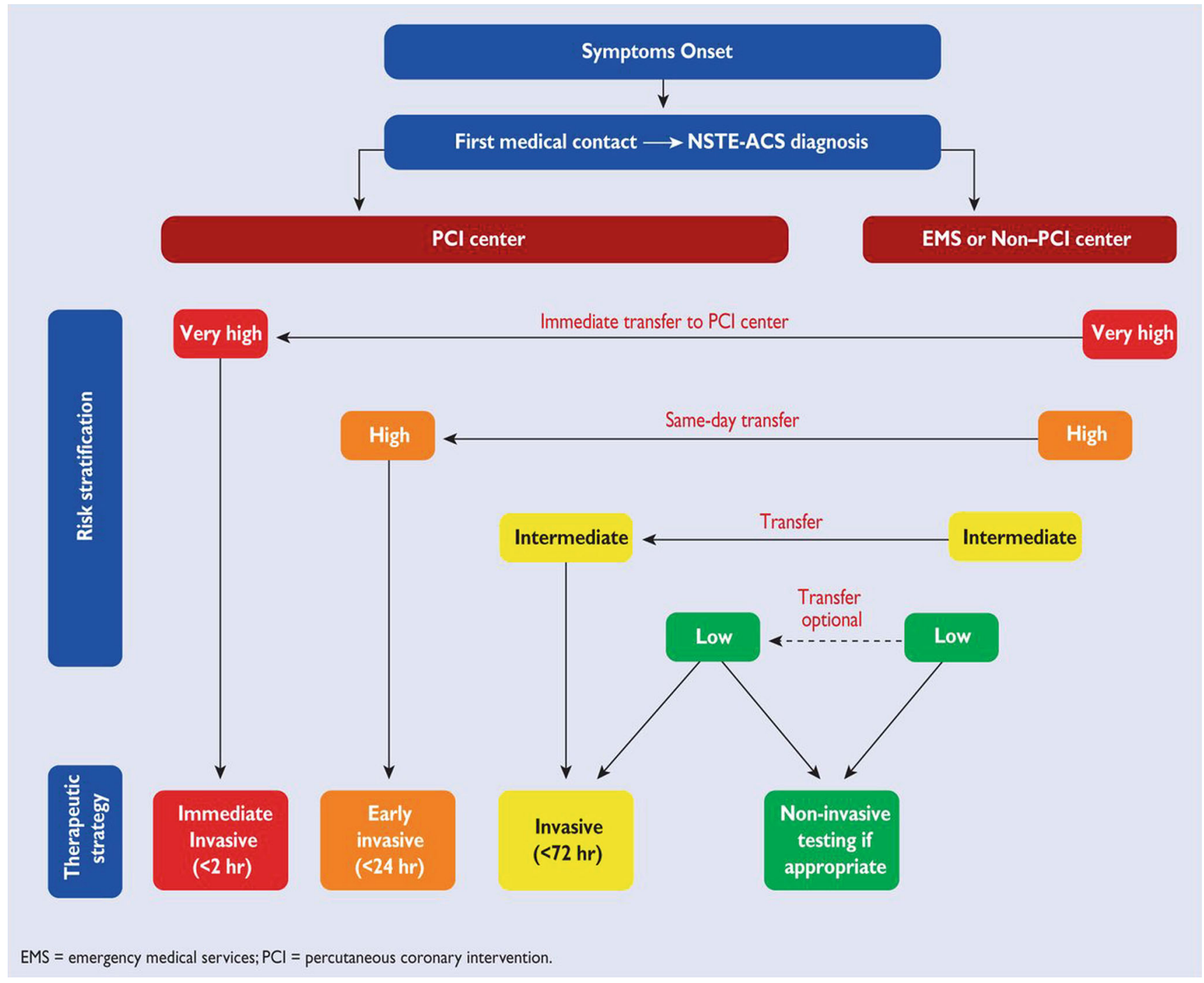

Fig. 4 Selection of non-ST-elevation acute coronary syndrome treatment strategy and timing according to initial risk stratification (With permission of Oxford University Press (UK)@ European Society of Cardiology, www.escardio.org)

sive coronary angiography is based on risk stratification and the assessment of the risks related to the procedure (Fig. 4).

\section{Very high-risk patients}

Patients at very high risk, including haemodynamic instability or cardiogenic shock, recurrent or ongoing chest pain refractory to medical treatment, life-threatening arrhythmias, mechanical complications of MI, acute heart failure, or recurrent dynamic ECG changes, should be referred for urgent PCI. Urgent PCI is defined as within $2 \mathrm{~h}$ of admission, analogous to primary PCI in ST-segment elevation MI.

\section{High-risk patients}

It is recommended that high-risk patients are transferred from a non-PCI centre to a PCI centre for coronary angiog- raphy within $24 \mathrm{~h}$. High-risk patients are defined as patients with a rise and fall in cardiac troponin comparable with MI, dynamic ST- or T-wave changes, or a GRACE score $>140$.

The ACS working group does not consider referral within $24 \mathrm{~h}$ to be a necessity for the Dutch situation, based on the following considerations. First, the scientific basis for the recommendation is weak as it is only based on two meta-analyses of randomised trials and a retrospective analysis of the ACUITY trial [7, 8]. Both meta-analyses showed no benefit for the hard endpoints mortality, nonfatal MI or major bleeding, but only a reduction in refractory ischaemia. Although the TIMACS trial demonstrated a beneficial effect of early intervention in a highrisk subgroup (GRACE >140), this was only a hypothesisgenerating result in a trial which did not show a significant reduction of the primary endpoint death or myocardial infarction [9]. Second, the Dutch situation is markedly 
different from that in many other European countries since the majority of Dutch cardiology departments are equipped with a catheterisation laboratory where diagnostic coronary angiography is routinely performed in ACS patients. After diagnostic angiography, patients are discussed in a heart team and only those patients suitable for PCI and CABG are referred to an interventional centre. We do not know whether referring all NSTEMI-ACS patients for undergoing catheterisation leads to over-treatment by performing adhoc PCI. Third, the current experience of non-PCI centres in the Netherlands as well as the results of the ICTUS trial show us that a more conservative (selective invasive) treatment of NSTE-ACS patients is also a good option [10]. Fourth, same-day transfer of patients based on a rise and fall in cardiac troponin might result in unnecessarily transferring patients with other pathology such as myocarditis or a type II MI (demand ischaemia) associated with heart failure of arrhythmias.

Other issues for implementing the 2015 ESC guidelines are that the Dutch hospitals and ambulance services do not have sufficient capacity for same-day transfer and that not performing the diagnostic angiogram in non-PCI centres could endanger the viability of the catheterisation laboratory in these hospitals. Subsequently, this might have important consequences for the role of the acute cardiac care and coronary care units and supply of patients. The ACS working group considers reducing catheterisation capacity in non-PCI centres not applicable if this is not based on proven-health benefits.

In this regard, from 2017, the ACS working group will inventorise and evaluate the current NSTE-ACS treatment in the Netherlands in collaboration with the Netherlands Society of Cardiology (NVVC), general practitioners, ambulance service, the NVVC ACS Connect project and the NCDR (national cardiovascular data registry). First results are expected in 2018.

Conflict of interest P. Damman, A.W. van 't Hof, J.M. ten Berg, J.W. Jukema, Y. Appelman, A.H. Liem and R.J. de Winter declare that they have no competing interests.

Open Access This article is distributed under the terms of the Creative Commons Attribution 4.0 International License (http:// creativecommons.org/licenses/by/4.0/), which permits unrestricted use, distribution, and reproduction in any medium, provided you give appropriate credit to the original author(s) and the source, provide a link to the Creative Commons license, and indicate if changes were made.

\section{References}

1. Roffi M, Patrono C, Collet JP, et al. 2015 ESC Guidelines for the management of acute coronary syndromes in patients presenting without persistent ST-segment elevation: Task Force for the Management of Acute Coronary Syndromes in Patients Presenting without Persistent ST-Segment Elevation of the European Society of Cardiology (ESC). Eur Heart J. 2016;37:267-315.

2. Mueller C. Biomarkers and acute coronary syndromes: an update. Eur Heart J. 2014;35:552-6.

3. Hamm CW, Bassand JP, Agewall S, et al. ESC Committee for Practice Guidelines. ESC Guidelines for the management of acute coronary syndromes in patients presenting without persistent STsegment elevation: The Task Force for the management of acute coronary syndromes (ACS) in patients presenting without persistent ST-segment elevation of the European Society of Cardiology (ESC). Eur Heart J. 2011;32:2999-3054.

4. ACCOAST Investigators, Montalescot G, Bolognese L, Dudek D, et al. Pretreatment with prasugrel in non-ST-segment elevation acute coronary syndromes. N Engl J Med. 2013;369:999-1010.

5. Hansen ML, Sørensen R, Clausen MT, et al. Risk of bleeding with single, dual, or triple therapy with warfarin, aspirin, and clopidogrel in patients with atrial fibrillation. Arch Intern Med. 2010;170:1433-41.

6. WOEST study investigators, Dewilde WJ, Oirbans T, Verheugt FW, et al. Use of clopidogrel with or without aspirin in patients taking oral anticoagulant therapy and undergoing percutaneous coronary intervention: an open-label, randomised, controlled trial. Lancet. 2013;381:1107-15.

7. Katritsis DG, Siontis GC, Kastrati A, et al. Optimal timing of coronary angiography and potential intervention in non-ST-elevation acute coronary syndromes. Eur Heart J. 2011;32:32-40.

8. Navarese EP, Gurbel PA, Andreotti F, et al. Optimal timing of coronary invasive strategy in non-ST-segment elevation acute coronary syndromes: a systematic review and meta-analysis. Ann Intern Med. 2013;158:261-70.

9. TIMACS Investigators, Mehta SR, Granger CB, Boden WE, et al. Early versus delayed invasive intervention in acute coronary syndromes. N Engl J Med. 2009;360:2165-75.

10. Damman P, Hirsch A, Windhausen F, Tijssen JG, de Winter RJ, ICTUS Investigators. 5-year clinical outcomes in the ICTUS (Invasive versus Conservative Treatment in Unstable coronary Syndromes) trial a randomized comparison of an early invasive versus selective invasive management in patients with non-ST-segment elevation acute coronary syndrome. J Am Coll Cardiol. 2010;55:858-64. 